\title{
COVID-19 and the Forgotten Majority
}

\author{
Massimo Volpe ${ }^{1,2} \cdot$ Giovanna Gallo $^{1}$ (D)
}

Received: 6 August 2020 / Accepted: 12 August 2020 / Published online: 29 August 2020

(c) Italian Society of Hypertension 2020

The SARS-CoV-2 pandemic and the related COVID-19 outbreak, accounting for more than 8 million cases worldwide, has represented and still represents a real earthquake for National Healthcare Systems in the planet. New intensive care units and wards specifically dedicated to this critical condition have been hurriedly opened and a change of destination of entire hospitals and departments was urgently planned and established [1]. Outpatient clinics were just shut down for months. Millions of visits, procedures, surgical operations and screening or prevention programs were cancelled or postponed through the world due to the unavoidable and necessary new mission of the hospitals to face the COVID-19 wave.

Indeed, hospital activities reserved for outpatients have been limited to urgent cases, the elective routine non-urgent activity being completely stopped in most cases. The general recommendation, shared by Centers for Disease Control and Prevention worldwide, has been to defer any test or procedure unlikely to directly impact on clinical care or outcomes [2,3].

An issue of particular importance is represented by the interruption of screening or follow-up programs, particularly in the context of cancer and cardiovascular diseases, leading to a worrying number of missed diagnoses and delayed specific treatments, necessary to prevent morbidity and mortality [1]. The management of patients with cancer or heart failure in more advanced stages will have a countless socioeconomic impact for healthcare systems and it will need an immediate new program to recover the missing actions as fast as possible.

Another relevant problem is represented by a substantial reduction of hospitalizations for acute conditions other than

Massimo Volpe

massimo.volpe@uniroma1.it

Giovanna Gallo

giovanna.gallo@uniroma1.it

1 Cardiology Unit, Department of Clinical and Molecular Medicine, Sant'Andrea Hospital, Sapienza University of Rome, Via di Grottarossa 1035, 00189 Rome, Italy

2 IRCCS Neuromed, Pozzilli, IS, Italy
COVID-19, such as acute coronary syndromes or cerebrovascular accidents. The patients' fear of a possible contagion in a hospital setting and the exclusive focus of emergency departments on COVID-19 with a consequent deferral of other procedures has generated an unpredicted pre-contemporary medical scenario $[4,5]$.

Several dramatic consequences of this sudden situation will face also the enormous burden of other missed medical actions during the COVID-19 crisis. In spite of the huge numerical impact of COVID, a much larger majority of patients with serious chronic diseases have lost the possibility to undergo planned follow-up visits, to be adequately monitored by referral physicians and to receive timely adjustment of therapeutic regimens.

Healthcare systems must rapidly react to give answers to a potentially "forgotten majority" and in this regard the growing use of telemedicine may provide a solid contribution.

\section{Declarations}

Conflict of interest On behalf of all authors, the corresponding author states that there is no conflict of interest.

\section{References}

1. The Lancet Oncology. Safeguarding cancer care in a postCOVID-19 world. Lancet Oncol. 2020;21(5):603. https://doi. org/10.1016/S1470-2045(20)30243-6.

2. 2020 ESC guidance for the diagnosis and management of CV disease in the context of the COVID-19 pandemic.

3. Driggin E, Madhavan MV, Bikdeli B, et al. Cardiovascular considerations for patients, health care workers, and health systems during the COVID-19 pandemic. J Am Coll Cardiol. 2020;75(18):2352-71. https://doi.org/10.1016/j.jacc.2020.03.031.

4. De Rosa S, Spaccarotella C, Basso C, et al. Reduction of hospitalizations for myocardial infarction in Italy in the COVID-19 era. Eur Heart J. 2020;41(22):2083-8. https://doi.org/10.1093/eurhe artj/ehaa409.

5. De Filippo O, D'Ascenzo F, Angelini F, et al. Reduced rate of hospital admissions for ACS during Covid-19 outbreak in northern Italy. N Engl J Med. 2020;383(1):88-9. https://doi.org/10.1056/ NEJMc2009166. 